\title{
An unforgettable debate between descriptive and experimental biology in the 1930s in China
}

\author{
Ming $\mathrm{Li}^{1}$, Zonggang $\mathrm{Hu}^{2}$, Le Kang ${ }^{1}$ \\ ${ }^{1}$ Beijing Institutes of Life Science, Chinese Academy of Sciences, Beijing 100101, China \\ 2 Lushan Botanical Garden, Jiangxi Province and Chinese Academy of Sciences, Jiujiang 332900, China
}

Erratum to: Protein Cell 2010, 1(12): 1053-1055

DOI 10.1007/s13238-010-0143-6

The photo in Figure 1 (Dr. Jingxi Wang) on the right column of page 1053 was incorrect. Presently the correct photo is unavailable.

The online version of the original article can be found at http://dx.doi.org/10.1007/s13238-010-0143-6 